\title{
Elevated Plasma X-Linked Neuroligin 4 Expression Is Associated with Autism Spectrum Disorder
}

\author{
Laila Y. Al-Ayadhia, b Hanan Y. Qasem ${ }^{a}$ Hend Ali M. Alghamdic \\ Nadra E. Elamin ${ }^{a}$ \\ ${ }^{a}$ Autism Research and Treatment Center, Faculty of Medicine, King Saud University, Riyadh, Saudi Arabia; \\ ${ }^{b}$ Department of Physiology, Faculty of Medicine, King Saud University, Riyadh, Saudi Arabia; 'College of Dentistry, \\ Princess Noura University, Riyadh, Saudi Arabia
}

\section{Highlights of the Study}

- This is the first study of a possible correlation between plasma neuroligin 4 (NLGN4) and autism severity.

- Increased plasma levels of NLGN4 suggest that it may play a role in the pathogenesis of autism.

- NLGN4 could possibly be a valuable biomarker for autism.

\section{Abstract}

Objectives: In this study, we compared plasma levels of neuroligin 4 (NLGN4) in children with autism versus matched healthy controls to examine a possible correlation between plasma NLGN4 and degree of autism severity as well as social impairment in autistic patients. Subjects and Methods: 88 autistic patients aged 3-12 years and 33 age- and sexmatched controls aged 3-9years were recruited. Plasma levels of NLGN4 were determined using a commercial enzymelinked immunoassay (ELISA). The Childhood Autism Rating Scale (CARS) and the Social Responsiveness Scale (SRS) were used to assess cognitive dysfunction and social impairment in autistic patients. Results: Plasma levels of NLGN4 were significantly higher $(p=0.001)$ in autistic children than in healthy controls. Despite alterations in the levels of NLGN4 in the subgroups of the autistic children, no correlation between plasma concentration of NLGN4 and cognitive problems or social impairment was observed ( $p>0.05)$. Conclusion: Increased plasma concentrations of NLGN4 may play a role in the pathogenesis of autism, and it could be a valuable biomarker for autism. Further studies with larger sample sizes are warranted to validate this finding and also to explore the potential links between NLGN4 and the features of autism.

(c) 2020 The Author(s) Published by S. Karger AG, Basel

\section{Introduction}

Autism is a common neurodevelopmental disorder characterized by impaired social interaction, communication problems, and stereotyped behaviors, together with sensory abnormalities [1,2]. The etiology of this disorder is still unresolved, with genetic and environmental factors implicated in the pathophysiology of autism $[1,3,4]$. The current estimated prevalence of autism is approximately $1 \%$, and the male-to-female ratio is $4: 1[5]$.

\section{karger@karger.com} www.karger.com/mpp Karger $\stackrel{\text { ' }}{=}$
(C) 2020 The Author(s) Published by S. Karger AG, Basel

Karger 0 pen access

This is an Open Access article licensed under the Creative Commons Attribution-NonCommercial-4.0 International License (CC BY-NC) (http://www.karger.com/Services/OpenAccessLicense), applicable to the online version of the article only. Usage and distribution for commercial purposes requires written permission. 
Neuroligins (NLGNs) are adhesion molecules that are expressed in postsynaptic neurons. They interact with neurexins (NRXNs) expressed in presynaptic neurons [6, 7]. Five genes encoding NLGN proteins have been identified in the human genome (NLGN1, NLGN2, NLGN3, NLGN4X, and NLGN4Y) whereas only NLGN1, NLGN2, NLGN3, and NLGN4 have been identified in other mammalian species like mice [8]. NLGNs are composed of an extended $\mathrm{N}$-terminal extracellular domain containing a large esterase homology domain, a short O-glycosylated region linked to a single transmembrane domain, and a short C-terminal intracellular domain (ICD) [9]. Several studies have suggested that levels of NLGNs are regulated by proteolytic processing; NLGNs are proteolytically cleaved in a sequential manner as a result of basal and also activity-dependent excitatory transmission $[10,11]$. NLGNs are initially cleaved by metalloproteases, which are responsible for the shedding of the ectodomain at the juxtamembrane stalk region, releasing the soluble form of NLGNs (sNLGNs) and a membrane-tethered C-terminal fragment (CTF). CTF then undergoes a subsequent cleavage mediated by $\gamma$-secretase to release the ICD. As proteolytic processing takes place on the cell surface, it leads to the removal of NLGNs from the cell surface and decreases their activity [10].

It has been hypothesized that the NLGN-NLXN interaction plays a role in synaptic function, formation, and stabilization. NLGN1, NLGN3, and NLGN4 are localized at excitatory glutamatergic axons, while NLGN2 is localized at inhibitory $\gamma$-aminobutyric acid (GABA) axons $[6,12]$.

Mutations of several synaptic proteins have been implicated in the molecular pathogenesis of neurodevelopmental disorders, including autism. These mutations may affect the synaptic pathway structure and function [13-15]. Mutations in NLGN3 and NLGN4 have been recorded in neurodevelopmental disorders [7, 16, 17].

The contribution of NLGNs to autism has been extensively examined at the genetic level. El-Kordi et al. [18] demonstrated high autism severity scores in an animal model involving NLGN4-null mutant mice. The severity of autism was represented as reduced social interaction, ultrasonic communication, altered aggression, enhanced selfgrooming and circling behavior, depression, anxiety, and tic disorders [15]. Children with autism exhibit significant cognitive and social impairments [19]. These impairments may be the most complex core challenge facing autistic patients [20]. The most common scales used for the assessment of the severity of autism and the associated social impairment are the Childhood Autism Rating Scale (CARS) and Social Responsiveness Scale (SRS), respectively [21,22].
This study was designed to evaluate the NLGN4 level in plasma samples of pediatric autistic patients versus healthy matched controls in an attempt to ascertain whether NLGN4 could be considered a biomarker for autism, and to examine the correlation between plasma NLGN4 level and autism severity and social impairment in autistic patients.

\section{Materials and Methods}

\section{Participants}

Eight-eight autistic children aged between 3 and 12 (5.5 \pm 2.2$)$ years were recruited from the Autism Research and Treatment Center, Faculty of Medicine, King Saud University, King Khalid University Hospital, Riyadh, Saudi Arabia. A clinical psychologist clinically diagnosed all autistic subjects, and the diagnosis was confirmed using the revised Autism Diagnostic Interview (ADI-R), the Autism Diagnostic Observation Schedule (ADOS), and the Developmental Dimensional Diagnostic Interview (3DI). Thirtythree age- and sex-matched control participants, aged between 3 and $9(5.3 \pm 1.2)$ years, were recruited from the Pediatric Clinic at the same hospital. Participants were excluded from the study if they had fragile X syndrome, serious neurological disorders, or any other medical conditions.

The severity of autism and the social impairment were determined using CARS and SRS, respectively [21, 22].

\section{Sample Collection}

Blood samples were drawn from 88 autistic children and 33 controls in the early morning after overnight fasting, and immediately collected into tubes containing EDTA. Blood samples were centrifuged at $4^{\circ} \mathrm{C}$ at $3,000 \mathrm{~g}$ for $20 \mathrm{~min}$. The plasma was decanted into different aliquots and stored at $-80^{\circ} \mathrm{C}$ until analysis.

\section{Biochemical Analysis}

Determination of NLGN4 Level

The plasma level of NLGN4 was measured using a commercial ELISA kit specific for NLGN4X (Cat. E6841h) according to the manufacturer's instructions (Wuhan EIAAb Science Inc., China). This immunoassay kit allows the in vitro quantitative determination of human NLGN4 concentrations in plasma. The microtiter plate provided in this kit was precoated with an antibody specific to NLGN4. Standards and samples were then added to the appropriate microtiter plate wells with a horseradish peroxidase (HRP)conjugated antibody and incubated. Substrate solutions were then added to each well. The enzyme-substrate reaction was terminated by the addition of a sulfuric acid solution, and the color change was measured spectrophotometrically at $450 \mathrm{~nm}$. The concentration of NLGN4 in the samples was then determined by comparing the optical density of the samples to the standard curve.

\section{Statistical Analyses}

The Statistical Package for Social Sciences v22 (SPSS, Chicago, IL, USA) was used. In all statistical analyses, an independent $t$ test with $p \leq 0.05$ was considered statistically significant. Statistical analysis was used to evaluate plasma NLGN4 levels in subjects diagnosed with autism versus controls. The correlation between the 
Table 1. Plasma NLGN4 level in the control and autistic groups, and CARS and SRS scores, and age in subjects with autism

\begin{tabular}{llrll}
\hline & Group & Mean & SD & $p$ value \\
\hline NLGN4, ng/mL & & & & \\
& controls $(n=33)$ & 6.821 & 5.521 & 0.001 \\
& autistic subjects $(n=88)$ & 12.299 & 5.348 & \\
\hline Autistic subjects & & & \\
CARS & mild-to-moderate $(n=53)$ & 12.881 & 5.091 & 0.271 \\
& severe $(n=35)$ & 11.417 & 5.676 & \\
SRS & mild-to-moderate $(n=22)$ & 13.469 & 4.839 & 0.213 \\
Age & severe $(n=66)$ & 11.909 & 5.486 & 0.492 \\
& $\leq 6$ years $(n=66)$ & 12.540 & 5.221 & \\
& $>6$ years $(n=22)$ & 11.575 & 5.777 & \\
\end{tabular}

NLGN4, neuroligin 4; CARS, Childhood Autism Rating Scale; SRS, Social Responsiveness Scale.

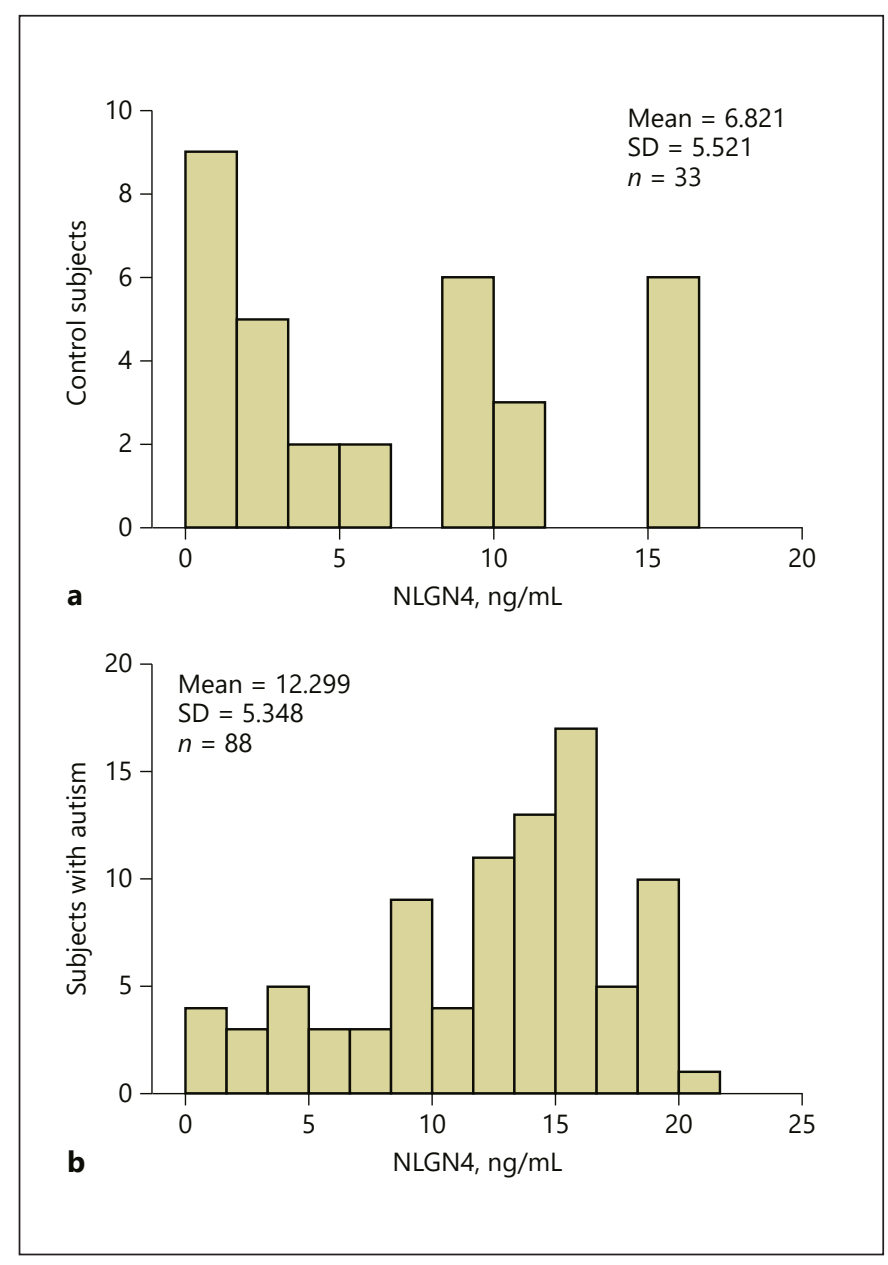

Fig. 1. Normal distribution of NLGN4 in controls (a) and subjects (b) with autism groups. NLGN4, neuroligin 4. level of NLGN4 and the risk of autism, presented as CARS and SRS scores, was evaluated to determine if there was a threshold level of NLGN4 above which the risk of having this disorder increases. The receiver-operating characteristics curve (ROC) was used to assess the specificity and sensitivity of NLGN4. ROC analysis was performed as a comprehensive way to measure the accuracy of the studied markers. The area under the curve (AUC) provides a useful metric to compare different biomarkers. An AUC value close to 1 indicates an excellent diagnostic and predictive marker whereas a curve that lies close to the diagonal (AUC 0.5) has no diagnostic utility. An AUC close to 1 is always accompanied by satisfactory specificity and sensitivity of the biomarker. Moreover, the predictiveness diagrams of the measured parameters were drawn, in which the $x$-axis represents the percentile rank of the biomarker, the $y$-axis the probability of identifying the disease, and the horizontal line the prevalence of the disease.

\section{Results}

Plasma concentration of NLGN4 was compared between children with autism and their age-matched controls (Table 1). The results showed a highly significant increase $(p=0.001)$ in plasma level of NLGN4 in the autistic children. Moreover, the subjects with autism were classified according to their CARS and SRS score, and their age, into different subgroups of autism severity (i.e., mild-to-moderate or severe). We observed that the level of NLGN4 in patients showing different degrees of cognitive and social impairment (CARS and SRS score, respectively) was increased in the mild-to-moderate autism subgroup compared to the severe autism subgroup, but the difference did not reach any statistical significance. Additionally, no significant difference between NLGN4 
Table 2. ROC analysis data of NLGN4 in the autistic group, CARS, SRS, and age in autistic children

\begin{tabular}{lcccc}
\hline & AUC & Best cutoff value & Sensitivity, \% & Specificity, \% \\
\hline $\begin{array}{l}\text { Patients with autism } \\
\text { CARS }\end{array}$ & 0.751 & 9.18 & 72.7 & 72.2 \\
$\quad$ Mild-to-moderate & 0.570 & 13.034 & 58.5 & 54.3 \\
$\quad$ Severe & 0.430 & 13.034 & 45.7 & 41.5 \\
SRS & & & \\
$\quad$ Mild-to-moderate & 0.573 & 13.49 & 59.1 & 51.5 \\
$\quad$ Severe & 0.427 & 13.66 & 47 & 40.9 \\
Age & & & 45.5 \\
$\quad \leq 6$ years & 0.544 & 12.513 & 56.1 & 0.439 \\
$\quad>6$ years & 0.456 & 13.034 & 0.455 & \\
\hline
\end{tabular}

AUC, area under the curve; CARS, Childhood Autism Rating Scale; SRS, Social Responsiveness Scale; ROC, receiver-operating characteristics; NLGN4, neuroligin 4.

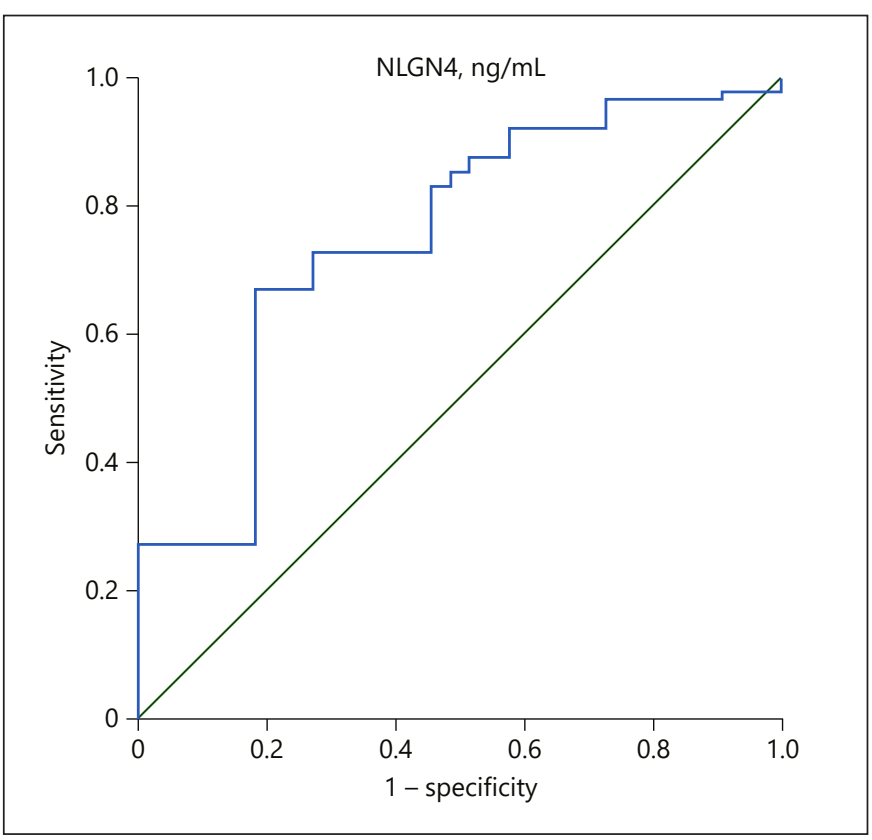

Fig. 2. ROC curve of NLGN4 $(\mathrm{ng} / \mathrm{mL})$ in autistic groups. ROC, receiver-operating curve; NLGN4, neuroligin 4.

level and age in subjects with autism was observed (Table 1). The individual distribution of data in autistic subjects compared to controls is presented in Figure 1.

Table 2 and Figure 2 demonstrate the ROC analysis presented as the AUC cutoff values for NLGN4 specificity and sensitivity in autistic subjects and in the subgroups. ROC curve analysis enables the best cutoff for clinical purposes. Sensitivity and specificity vary with the cutoff chosen for the test and depend upon the clinical context of the research. This study indicated an AUC of around 0.751, which is considered a satisfactory value of accuracy with a high specificity and sensitivity (72.2 and $72.7 \%$, respectively). The best cutoff value obtained from the ROC analysis was $9.18 \mathrm{ng} / \mathrm{mL}$. NLGN4 showed good specificity and sensitivity but not good enough for clinical purposes. Table 2 also demonstrates that NLGN4 in the patient subgroup exhibited an AUC value close to 0.5 which is considered to be poor.

\section{Discussion}

This is the first study to determine the plasma level of NLGN4 in autism; all previous studies were done at the genetic level. There was a significantly increased level of plasma NLGN4 in autistic subjects compared to matched controls, suggesting that NLGN4 may play a role in the pathogenesis of autism. Furthermore, the ROC curve results confirmed that NLGN4 may be considered for use as a risk biomarker for autism.

Although most of the previous experimental studies on nervous system disease biomarkers were performed on tissues or whole-blood samples, studying their level in the plasma as a complex body fluid can reflect pathophysiological activity in the central nervous system. Since $500 \mathrm{~mL}$ of cerebrospinal fluid in the human body is absorbed into the blood every day, this makes plasma a good source of biomarkers of diseases of the nervous system [23].

Genetic studies of autism have revealed various numbers of gene mutations in synaptic proteins that are im- 
plicated in autism, leading to synaptic dysfunction and causing a wide range of behavioral impairments and social interaction deficits [15, 24-26].

NLGN4 is known to play a critical role in synaptic function, formation, and stabilization, so the impaired NLGNs may lead to an excitatory/inhibitory imbalance and a selective loss of the inhibitory function of neurons $[9,27,28]$. In addition, the relationship between glutamate, GABA and the NLGN family has been established in autism. It has been shown that NLGNs are localized at both glutamatergic and GABAergic synapses in the hippocampal neurons. Alterations in glutamate and GABA levels in samples from autism sufferers have been shown to affect NLGN function in synapse stabilization $[28,29]$. Taken together, the higher level of NLGN4 reported in this study may be a consequence of synaptic dysfunction associated with autism, or else due to alterations in other components involved in synaptogenesis. However, these data should be treated with caution until further studies with a larger sample size are performed to validate these results and elucidate the mechanism by which NLGN4 expression may contribute to the pathogenesis of autism.

Chih et al. [30] showed that a nonsense mutation in NLGN4 in hippocampal neurons caused retention of NLGN4 protein in the endoplasmic reticulum. They also concluded that mutant NLGN4 protein failed to promote presynaptic differentiation, and hence leads to complete functional inactivation. Another study carried out by Talebizadeh et al. [17] suggested that genetic alteration other than mutations such as splice variants may lead to abnormal function of NLGN4 in autism spectrum disorder. These findings may explain the higher plasma level of NLGN4 that we observed, suggesting that mutations may lead to increased production of NLGN4 but without any functional effect.

Although there was no correlation between NLGN4 and CARS or SRS observed among the subgroups of autistic subjects, it seems that NLGN4 alteration may affect the cognitive and social behaviors in some subgroups of patients [14]. This could be due to an inadequate sample size that failed to detect significant changes between NLGN4 and the behavioral scores measured.

One of the limitations of our study was the small sample size. The lack of correlation between the plasma levels of NLGN4 and cognitive problems or social impairment could be attributed to the sample size, as the statistical power failed to detect significant changes between the studied parameters. A further potential limitation is the lack of previous research into plasma
NLGN4; we were unable to assess the effect of NLGN4 on autism severity. Therefore, there is a need for similar but larger-scale studies involving plasma. This would fill the gap in the literature and help us to understand the potential links between NLGN4 and cognition and social impairment.

\section{Conclusion}

This study highlights the possible relevance of plasma NLGN4 in the etiology of autism. The ROC curve suggests that NLGN4 could be used as a risk biomarker for autism. Future studies ought to focus on the exact role of NLGN4 in the pathophysiology of autism. Furthermore, interventional studies are recommended to explore the potential links between NLGN4 and features of autism.

\section{Acknowledgment}

The project was funded by the National Plan for Science, Technology and Innovation (MAARIFAH), King Abdulaziz City for Science and Technology, Kingdom of Saudi Arabia, Award No. (08-MED 510-02).

\section{Statement of Ethics}

This study was approved by the Medical Ethics Committee of King Saud University, according to the most recent Declaration of Helsinki principles. Parents provided informed consent prior to their children's participation in the study according to the guidelines of the Ethics Committee of King Saud University, King Khalid University Hospital.

\section{Disclosure Statement}

The authors declare that they have no conflicts of interest.

\section{Author Contributions}

L.Y.A.-A.: designed the study, conducted the study, and codrafted the manuscript. H.Y.Q.: performed data analysis and drafted the manuscript. H.A.M.A.: carried out the biochemical assays and literature review. N.E.E.: codrafted and revised the manuscript. All authors read and approved the final manuscript. 


\section{References}

1 Chaste P, Leboyer M. Autism risk factors: genes, environment, and gene-environment interactions. Dialogues Clin Neurosci. 2012 Sep;14(3):281-92.

2 American Psychiatric Association. Diagnostic and statistical manual of mental disorders. 4th ed., text revision. Washington: D.C American Psychiatric Association; 2013.

3 Bjorklund G, Saad K, Chirumbolo S, Kern JK, Geier DA, Geier MR, et al. Immune dysfunction and neuroinflammation in autism spectrum disorder. Acta Neurobiol Exp (Warsz). 2016;76(4):257-68.

4 Muhle R, Trentacoste SV, Rapin I. The genetics of autism. Pediatrics. 2004 May;113(5):e472-86.

5 Elsabbagh M, Divan G, Koh YJ, Kim YS, Kauchali S, Marcín C, et al. Global prevalence of autism and other pervasive developmental disorders. Autism Res. 2012 Jun;5(3):160-79.

6 Jamain S, Quach H, Betancur C, Råstam M, Colineaux C, Gillberg IC, et al.; Paris Autism Research International Sibpair Study. Mutations of the X-linked genes encoding neuroligins NLGN3 and NLGN4 are associated with autism. Nat Genet. 2003 May;34(1):27-9.

7 Boucard AA, Chubykin AA, Comoletti D, Taylor P, Südhof TC. A splice code for transsynaptic cell adhesion mediated by binding of neuroligin 1 to $\alpha$ - and $\beta$-neurexins. Neuron. 2005 Oct; 48(2):229-36.

8 Bolliger MF, Frei K, Winterhalter KH, Gloor $\mathrm{SM}$. Identification of a novel neuroligin in humans which binds to PSD-95 and has a widespread expression. Biochem J. 2001 Jun; 356(Pt 2):581-8.

9 Chih B, Engelman H, Scheiffele P. Control of excitatory and inhibitory synapse formation by neuroligins. Science. 2005 Feb;307(5713): 1324-8.

10 Suzuki K, Hayashi Y, Nakahara S, Kumazaki H, Prox J, Horiuchi K, et al. Activity-dependent proteolytic cleavage of neuroligin-1. Neuron. 2012 Oct;76(2):410-22.

11 Bemben MA, Nguyen TA, Li Y, Wang T, Nicoll RA, Roche KW. Isoform-specific cleavage of neuroligin-3 reduces synapse strength. Mol Psychiatry. 2019 Jan;24(1):145-60.
12 Arons MH, Thynne CJ, Grabrucker AM, Li D, Schoen M, Cheyne JE, et al. Autism-associated mutations in ProSAP2/Shank3 impair synaptic transmission and neurexin-neuroliginmediated transsynaptic signaling. J Neurosci. 2012 Oct;32(43):14966-78.

13 Shi L, Chang X, Zhang P, Coba MP, Lu W, Wang $\mathrm{K}$. The functional genetic link of NLGN4X knockdown and neurodevelopment in neural stem cells. Hum Mol Genet. 2013 Sep; 22(18):3749-60.

14 Südhof TC. Neuroligins and neurexins link synaptic function to cognitive disease. $\mathrm{Na}$ ture. 2008 Oct;455(7215):903-11.

15 Jaramillo TC, Liu S, Pettersen A, Birnbaum SG, Powell CM. Autism-related neuroligin-3 mutation alters social behavior and spatial learning. Autism Res. 2014 Apr;7(2):264-72.

16 Laumonnier F, Bonnet-Brilhault F, Gomot M, Blanc R, David A, Moizard MP, et al. Xlinked mental retardation and autism are associated with a mutation in the NLGN4 gene, a member of the neuroligin family. Am J Hum Genet. 2004 Mar;74(3):552-7.

17 Talebizadeh Z, Lam DY, Theodoro MF, Bittel DC, Lushington GH, Butler MG. Novel splice isoforms for NLGN3 and NLGN4 with possible implications in autism. J Med Genet. 2006 May;43(5):e21

18 El-Kordi A, Winkler D, Hammerschmidt K, Kästner A, Krueger D, Ronnenberg A, et al. Development of an autism severity score for mice using Nlgn4 null mutants as a constructvalid model of heritable monogenic autism. Behav Brain Res. 2013 Aug;251:41-9.

19 Eaves LC, Ho HH. Young adult outcome of autism spectrum disorders. J Autism Dev Disord. 2008 Apr;38(4):739-47.

20 Yang DY, Baillargeon R. Brief report: difficulty in understanding social acting (but not false beliefs) mediates the link between autistic traits and ingroup relationships. J Autism Dev Disord. 2013 Sep;43(9):2199-206.

21 Schopler E, Reichler RJ, Renner BR. The childhood autism rating scale (CARS). CA: Western Psychological Services Los Angeles; 2002 .
22 Constantino JN, Davis SA, Todd RD, Schindler MK, Gross MM, Brophy SL, et al. Validation of a brief quantitative measure of autistic traits: comparison of the social responsiveness scale with the autism diagnostic interview-revised. J Autism Dev Disord. 2003 Aug;33(4):427-33.

23 Hye A, Lynham S, Thambisetty M, Causevic M, Campbell J, Byers HL, et al. Proteomebased plasma biomarkers for Alzheimer's disease. Brain. 2006 Nov;129(Pt 11):3042-50.

24 Zhang C, Milunsky JM, Newton S, Ko J, Zhao $\mathrm{G}$, Maher TA, et al. A neuroligin-4 missense mutation associated with autism impairs neuroligin-4 folding and endoplasmic reticulum export. J Neurosci. 2009 Sep;29(35):1084354.

25 Rothwell PE, Fuccillo MV, Maxeiner S, Hayton SJ, Gokce O, Lim BK, et al. Autism-associated neuroligin-3 mutations commonly impair striatal circuits to boost repetitive behaviors. Cell. 2014 Jul;158(1):198-212.

26 Hosie S, Ellis M, Swaminathan M, Ramalhosa F, Seger GO, Balasuriya GK, et al. Gastrointestinal dysfunction in patients and mice expressing the autism-associated R451C mutation in neuroligin-3. Autism Res. 2019 Jul; 12(7):1043-56.

27 Chanda S, Aoto J, Lee SJ, Wernig M, Südhof TC. Pathogenic mechanism of an autism-associated neuroligin mutation involves altered AMPA-receptor trafficking. Mol Psychiatry. 2016 Feb;21(2):169-77.

28 Zhang B, Gokce O, Hale WD, Brose N, Südhof TC. Autism-associated neuroligin-4 mutation selectively impairs glycinergic synaptic transmission in mouse brainstem synapses. J Exp Med. 2018 Jun;215(6):1543-53.

29 Blatt GJ, Fatemi SH. Alterations in GABAergic biomarkers in the autism brain: research findings and clinical implications. Anat Rec (Hoboken). 2011 Oct;294(10):1646-52.

30 Chih B, Afridi SK, Clark L, Scheiffele P. Disorder-associated mutations lead to functional inactivation of neuroligins. Hum Mol Genet. 2004 Jul;13(14):1471-7.
Plasma Neuroligin 4 and Autism

Spectrum Disorder
Med Princ Pract 2020;29:480-485

DOI: $10.1159 / 000507081$ 